\title{
ECONOMETRIC THEORY
}

\section{Notes for Contributors}

Contributions. Contributions are welcomed from all countries. They should be written in English.

Manuscripts. Manuscripts should be submitted electronically via Editorial Express at

https://editorialexpress.com/et

Files should be in PDF format with all fonts (and graphics) embedded and should be accompanied by a letter of submission.

Manuscripts are accepted for review on the understanding that the same work has not been and will not be published nor is presently submitted elsewhere. While under editorial review, it is the responsibility of the authors to keep the Editor informed about submissions, publication plans, and actual publication of related research or abstracts thereof in other outlets, including letters, journals, review publications, journals in other disciplines, conference proceedings, and published dissertations. It is further understood that all persons listed as authors have given their approval for the submission of the article and that any person cited as a source of personal communication has approved such citation; written authorization may be required at the Editor's discretion. Authors are responsible for obtaining written permission to publish material for which they do not own the copyright. Articles and other material published in Econometric Theory represent the opinions of the authors and should not be construed to reflect the opinions of the Editor, Advisory Board, Editorial Board, or the Publisher.

Manuscript Revision. In the event that a manuscript is returned to the author for revision, the revised Manuscript should normally be submitted no longer than one year after the revision request, unless otherwise indicated by the Co-Editor. A Revision submitted after this time will generally be treated as a new submission.

File Format for Accepted Manuscripts. Submissions of all accepted manuscripts should include both a PDF file of the complete final version of the article together with all source files derived from any TeX program (Latex, Scientific Word) and any graphics files.

Preparation of Accepted Manuscripts. The entire manuscript (including notes and references) should be typed in 10 pt. or larger, at least 1.5 (preferably double) spacing, with wide margins to accommodate copyediting. Manuscript pages should be numbered consecutively. Page 1 should provide article title, author name(s) in the form preferred for publication, complete affiliation(s), and e-mail address(es). At the bottom of Page 1 place any footnotes to the title or authors, indicated by superscripts *, **, etc. Page 2 should contain a proposed running head (abbreviated title of no more then 40 characters) and the name of the author to whom proofs should be sent. Page 2 should also contain a short abstract of the article in less than 150 words. The Abstract will appear at the head of the article when published in the Journal.

Theorem, lemma, proposition, and definition statements should appear in italic print.

Equations. All equations should be typewritten and the numbers for displayed equations should be in parentheses in the right margin [e.g., (1), (2), etc.; for Appendix equations use (A.1), (A.2), etc.]. Text mentions of equations should simply use the form (2) or (A.3). Superscripts and subscripts should be typed clearly above and below the line, respectively. End-of-proof signposts should appear as $\boldsymbol{\square}$. The mathematical expectation operator should be set in blackboard bold (open faced) E. Authors are encouraged to use the following order for enclosures, $\{[(\ldots)]\}$.

Tables and Figures. Tables and figures should be numbered consecutively in the order in which they are mentioned in the text. Every table or figure should have a title or caption and at least one reference in the text to indicate its appropriate location. Figures must be submitted ready for reproduction in electronic form, preferably TIFF (line drawings at least 600 dpi, grey scale at least $300 \mathrm{dpi}$ ) or EPS (with fonts embedded) format. Figures should be submitted as high-resolution files. TIFF, EPS, or PDF files must be provided for all figures. Figures should remain legible at a $50 \%$ reduction, and letters within a word should not touch one another. Figure labels should correspond to text notation as to italic or roman typeface, and superscripts and subscripts should be in superior and inferior positions.

References. First text citation of each reference should include all authors' last names (and the year); use "et al." thereafter. Complete bibliographic information for each citation should be included in the list of references. Journal names should not be abbreviated. References should be in alphabetical order and presented in the style of the following examples:

Bergstrom, A.R. (1976) Statistical Inference in Continuous Time Economic Models. North-Holland.

Giraitis, L. \& D. Surgailis (1986) Multivariate Appell polynomials and the central limit theorem. In E. Eberlein \& M.S. Taqqu (eds.), Dependence in Probability and Statistics: A Survey of Recent Results, pp. 21-72. Birkhauser.

Gregory, A.W. \& G.W. Smith (1995) Business cycle theory and econometrics. Economic Journal 105, 1597-1608.
Wooldridge, J.M. (in press) On the limits of GLM for specification testing: A comment on Gurmu and Trivedi. Econometric Theory.

Footnotes. When more than a simple source citation is called for, footnotes may be used. These should be numbered consecutively throughout the article and listed together at the end of the text, before the references. Source citations within footnotes follow the same style as citations in the tex

Copyediting and Proofreading. The publisher reserves the right to copyedit and proofread all articles accepted for publication, but authors will be asked to review their manuscripts if changes have been substantial. Proofs will be sent (as PDF files attached to e-mail) to the corresponding author for corrections of typographical errors only.

Offprints. Authors may purchase offprints if they are ordered at the proof stage (an order form will be sent with proofs).

EDITORIAL POLICY. Since its inception, Econometric Theory has aimed to endow econometrics with an innovative journal dedicated to advance theoretical research in econometrics. It provides a centralized professional outlet for original theoretical contributions in all of the major areas of econometrics, and all fields of research in econometric theory fall within the scope of ET. In addition, ET fosters the multidisciplinary features of econometrics that extend beyond economics. Particularly welcome are articles that promote original econometric research in relation to mathematical finance, stochastic processes, statistics, and probability theory, as well as computationally intensive areas of economics such as modern industrial organization and dynamic macroeconomics. Contributions that exposit methodological and technical advances in these fields and that illustrate their potential in econometric research are actively encouraged. Articles that unify earlier econometric work either in productive ways or by the use of more elegant methods lie within the scope of the Journal. In recognition of the interface between theory and practice in modern econometric research, ET encourages submissions that promote best practice econometrics by demonstrating new theory in conjunction with the practical implementation of theory.

As well as articles that embody original theoretical research, ET publishes historical studies on the evolution of econometric thought and interviews with the subject's leading scholars. 


\section{ARTICLES}

\section{Markku Lanne and Pentti Saikkonen}

Noncausal Vector Autoregression

\section{Dong Li, Shiqing Ling, and Wai Keung Li}

Asymptotic Theory on the Least Squares Estimation of Threshold

Moving-Average Models

Robert P. Lieli and Maxwell B. Stinchcombe

On the Recoverability of Forecasters' Preferences

Marco Avarucci, Eric Beutner, and Paolo Zaffaroni

On Moment Conditions for Quasi-Maximum Likelihood Estimation of Multivariate ARCH Models

Raffaella Giacomini, Dimitris N. Politis, and Halbert White

A Warp-Speed Method for Conducting Monte Carlo Experiments Involving Bootstrap Estimators

$$
\text { ET INTERVIEW }
$$

\section{Joris Pinkse}

ET Interview with Herman Bierens

\section{MISCELLANEA}

\section{Timothy J. Vogelsang and Martin Wagner}

A Fixed- $b$ Perspective on the Phillips-Perron Unit Root Tests

\section{Cong Li and Jeffrey S. Racine}

A Smooth Nonparametric Conditional Density Test for Categorical Responses

\section{Benedikt M. Pötscher}

On the Order of Magnitude of Sums of Negative Powers of Integrated Processes

Yiguo Sun, Zongwu Cai, and Qi Li

Semiparametric Functional Coefficient Models with Integrated Covariates

Cambridge Journals Online

For further information about this journal

please go to the journal website at:

journals.cambridge.org/ect

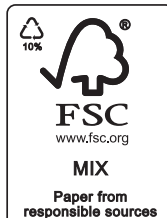

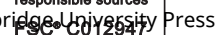

CAMBRIDGE UNIVERSITY PRESS 